\title{
On $t$-fold covers of coherent configurations
}

\author{
Alyssa D. Sankey \\ Department of Mathematics \& Statistics, University of New Brunswick, \\ P.O. Box 4400, Fredericton, N.B., E3B 5A3, Canada
}

Received 15 September 2015, accepted 27 June 2017, published online 29 October2017

\begin{abstract}
We introduce the covering configuration induced by a regular weight defined on a coherent configuration. This construction generalizes the well-known equivalence of regular two-graphs and antipodal double covers of complete graphs. It also recovers, as special cases, the rank 6 association schemes connected with regular 3-graphs, and certain extended Q-bipartite doubles of cometric association schemes. We articulate sufficient conditions on the parameters of a coherent configuration for it to arise as a covering configuration.
\end{abstract}

Keywords: Association scheme, coherent configuration, regular weight, double cover, two-graph, t-graph.

Math. Subj. Class.: 05C22, 05C50, 05E30

\section{Introduction}

The Seidel matrix of a graph $\Gamma$ may be viewed as a weight on the complete graph: edges of $\Gamma$ are weighted $(-1)$ and non-edges $(+1)$. If $\Gamma$ is strongly regular with $n=2(2 k-\lambda-\mu)$, it lies in the switching class of a regular two-graph and we call the weight, analogously, regular on $K_{n}$. This condition on $\Gamma$ is well known, and dates to 1977, in [25]. The same year, the equivalence of regular two-graphs and antipodal double covers of complete graphs was established in [26].

Martin, Muzychuk and Williford ([18]) defined the extended Q-bipartite double of a cometric association scheme, extending the notion of the bipartite double of a distance regular graph. This construction produces, as special cases, the antipodal double covers of complete graphs from the strongly regular graphs affording regular two-graphs.

In recent work, Kalmanovich ([16]) has also generalized the regular two-graph result, working from an unpublished draft of D. G. Higman's ([9]) on regular 3-graphs. As defined in [14], a $t$-graph weights the edges of $K_{n}$ with elements of the group of roots of unity of

E-mail address: asankey@unb.ca (Alyssa D. Sankey)

(a) (i) This work is licensed under http://creativecommons.org/licenses/by/3.0/ 
order $t, U_{t}$. The regularity condition ensures that the matrix of edge weights has a quadratic minimal polynomial. The work of Kalmanovich-Higman establishes the equivalence of regular 3-graphs with cyclic antipodal 3-fold covers of $K_{n}$ ([6]). Regular 3-graphs are shown to give rise to certain rank 6 association schemes, and the necessary conditions under which a rank 6 scheme arises in this way are given.

In this paper there are two main results. First, working with a regular weight with values in $U_{t}$, defined on a coherent configuration (CC), we show that there is always a covering configuration; that is, a CC constructed using a $t$-fold cover in a natural way, to convert the weight into a $\mathrm{CC}$ of higher rank (by a factor of $t$ ). As special cases, we recover the equivalence between regular two-graphs and antipodal double covers of complete graphs; some extended Q-bipartite doubles of cometric schemes; the rank 6 schemes associated with regular 3-graphs, and an extension of these to regular $t$-graphs.

A CC with a regular weight has two sets of parameters: the structure constants for the weighted adjacency algebra, $\left\{\beta_{i j}^{k}\right\}$, which lie in $\mathbb{C}$ or more specifically in the ring of integers with a primitive $t^{\text {th }}$ root of unity adjoined, and the non-negative integers $\left\{\beta_{i j}^{k}(\nu)\right\}$ which count certain triangles with a specified weight. They are related by

$$
\beta_{i j}^{k}=\sum_{\nu \in U_{t}} \nu \beta_{i j}^{k}(\nu) .
$$

The weighted adjacency algebra is in general not a coherent algebra, and may in fact have a coherent closure that is much higher in rank than the original CC. In the regular two-graph case, for instance, it is precisely when the $(-1)$ edges form an SRG that we get a minimal closure: a natural fission of the edge set into $(+1)$ and $(-1)$ edges that yields a (rank 3 ) association scheme. The covering configuration is the realization of a CC whose structure constants are the $\beta_{i j}^{k}(\nu)$. Some properties, namely homogeneity and commutativity of a $\mathrm{CC}$ carry over to the covering configuration. Symmetry is preserved only if $t=2$. Metric and cometric properties are not.

The second main result of this paper is the articulation of sufficient conditions for a CC to be the covering configuration of a regular weight.

In the final section, we describe a family of regular weights on the Hamming Scheme $H(n, 2)$ with values in $U_{4}$, due to Ada Chan. These weights all fuse to regular 4-graphs, providing an infinite family that may be of interest as complex Hadamard matrices. These regular weights, and their fusions, admit covering configurations of ranks $4(n+1)$ and 8 respectively, on $2^{n+2}$ points.

\section{Preliminaries}

In this section, we give the definitions that are essential to what follows. Much more can be found in [17] and in the original developments of the area by Weisfeiler and Lehman in [28] and by D. G. Higman in [11, 12], and [14].

\subsection{Coherent configurations}

Definition 2.1. Let $\left\{A_{i}\right\}_{0 \leq i<r}$ be a set of 01-matrices with rows and columns indexed by a finite set $X$. Let $\mathcal{I}:=\{0,1, \ldots, r\}$. The linear $\operatorname{span} \mathcal{A}:=\left\langle A_{i}\right\rangle_{\mathbb{C}}$ is a coherent algebra if:

(i) $\sum_{i \in \mathcal{I}} A_{i}=J$, where $J$ is the all-ones matrix,

(ii) $\sum_{i \in \mathcal{L}} A_{i}=I$, for some subset $\mathcal{L} \subset \mathcal{I}$, 
(iii) for each $i$ there exists $i^{*} \in \mathcal{I}$ such that $A_{i}^{T}=A_{i^{*}}$,

(iv) $A_{i} A_{j}=\sum p_{i j}^{k} A_{k}, p_{i j}^{k} \in \mathbb{Z}^{+}$.

A coherent algebra (CA), is homogeneous if $|\mathcal{L}|=1$; symmetric if $i^{*}=i$ for all $i$, and commutative, clearly, if $p_{i j}^{k}=p_{j i}^{k}$ for all $i, j, k$. The homogeneous CAs are (possibly non-symmetric) association schemes. Commutative schemes which have the metric or P-polynomial property are synonymous with distance-regular graphs (DRGs); those of diameter 2 are the strongly regular graphs (SRGs). Some familiarity with these structures is assumed. References for readers lacking this background are [1, 2, 4, 5, 19], and [27]. In the association scheme literature, a rank $r$ scheme is often referred to as an $(r-1)$-class scheme: 'rank' counts the trivial relation, while the number of 'classes' does not.

Every algebra of $n$ by $n$ matrices over $\mathbb{C}$ that is closed under transpose and entry-wise multiplication, and contains both $I$ and $J$ is a coherent algebra, and as such it has a basis of 01-matrices satisfying (i)-(iv). Each $A_{i}$ in a CA is the adjacency matrix of a digraph $\Gamma_{i}$ with vertex set $X$, which is simple for $i \notin \mathcal{L}$ and a graph when $i^{*}=i$. Viewing these graphs as relations on $X$, define a coherent configuration (CC) to be a set of binary relations on $X$, indexed by $\mathcal{I}$, with analogous properties to (i)-(iv) above. Denote it $\mathfrak{A}:=\left(X,\left\{R_{i}\right\}_{i \in \mathcal{I}}\right)$.

The constant $p_{i j}^{k}$ counts the number of $i$ - $j$ paths from a vertex $x$ to a vertex $z$, given that $(x, z) \in R_{k}$ and this number is necessarily independent of the choice of edge in $\Gamma_{k}$. It is convenient to denote each instance of an $i$ - $j$ path by a triangle $(x, y, z)$ of type $(i, j, k)$. That is, $(x, y, z) \in X^{3}$ is a triangle of type $(i, j, k)$ if $(x, y) \in R_{i},(y, z) \in R_{j}$, and $(x, z) \in R_{k}$ as indicated in Figure 1 .

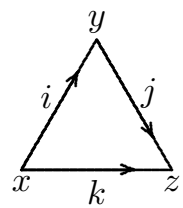

Figure 1: Triangle $(x, y, z)$ of type $(i, j, k)$.

Define the intersection matrices $M_{j}$ of a CC by $M_{j}:=\left(p_{i j}^{k}\right), 0 \leq i, k<r$ thus the map

$$
\gamma: A_{j} \mapsto M_{j}
$$

is the right regular representation of $\mathcal{A}$.

We treat CAs and CCs as equivalent structures and move freely between the notations of matrices, relations, and graphs. As $\left\{A_{i}\right\}$ forms the standard basis of $\mathcal{A}$, we refer to $\left\{R_{i}\right\}$ and $\left\{\Gamma_{i}\right\}$ as the basic relations and basic graphs of $\mathfrak{A}$ respectively.

\subsection{Fusion and fission}

A fusion is a merging of relations in a $\mathrm{CC}$ according to a partition of $\mathcal{I}$. A fusion will be deemed coherent if the resulting configuration is coherent. A coherent fission or refinement is a partition of each basic relation such that the resulting set of relations forms a CC. 
The rank $2 \mathrm{CC}$ represented by $K_{n}$ is the minimum element in the lattice of all CCs on a given vertex set $X$ of size $n$ ([12, Prop. 3]). The maximum element has rank $n^{2}$, with the full matrix algebra $M_{X}(\mathbb{C})$ as its coherent algebra.

\subsection{Regular weights}

Let $U=U_{t}$ be the group of complex $t^{\text {th }}$ roots of unity, and fix a primitive $\operatorname{root} \zeta$ as the generator of $U$.

Definition 2.2. A weight with values in $U$ is a 2-cochain $\omega: X^{2} \rightarrow U$. Viewed as a matrix, a weight is Hermitian with unit diagonal.

The coboundary of $\omega$ is a function on triangles:

$$
\delta \omega(x, y, z):=\omega(y, z) \overline{\omega(x, z)} \omega(x, y)
$$

and we refer to this value as the weight of the triangle $(x, y, z)$. Analogous to Seidel switching on a graph, switching a weight $\omega$ at vertex $x_{i}$ by a factor of $\alpha \in U$ multiplies the weight on $\left(x_{i}, y\right)$ edges by $\alpha$ and on $\left(y, x_{i}\right)$ edges by $\bar{\alpha}$ for all $y \neq x_{i}$. In matrix form, this is a similarity transform by the diagonal matrix $\operatorname{diag}(1,1, \ldots, 1, \alpha, 1, \ldots, 1)$ with $\alpha$ in position $i$. We refer to two weights as switching equivalent if one is obtained from the other by some sequence of switches, and observe that $\delta \omega$ is invariant under switching.

Definition 2.3. A t-graph is $\delta \omega$ for some weight $\omega$. It is regular if

$$
|\{y \mid \delta \omega(x, y, z)=\alpha\}|
$$

is independent of $x$ and $z$, for each value $\alpha \in U$.

This is one of a number of natural generalizations of the regular two-graph ([9, 16, 22, $23,24,25])$. Since a 2-cochain is equivalent to a weight on the edges of a complete graph, the notion of regularity can be extended to weights on CAs.

The entry-wise product $\omega \circ A_{i}$ gives a matrix with $(x, y)$ entry equal to $\omega(x, y)$ where $(x, y) \in R_{i}$. Denote this weighted adjacency matrix $A_{i}^{\omega}$.

Definition 2.4 ([14]). A weight $\omega$ is regular on a CC if for $(x, z) \in R_{k}$ the number of triangles $(x, y, z)$ of type $(i, j, k)$ and weight $\alpha$ is independent of $x$ and $z$. In this case, the number of such triangles depends on $i, j, k$, and $\alpha$ and we denote this parameter $\beta_{i j}^{k}(\alpha)$.

If $\omega$ is regular on $\mathfrak{A}$, then $\sum_{\alpha} \beta_{i j}^{k}(\alpha)=p_{i j}^{k}$. By a straight-forward counting argument,

$$
A_{i}^{\omega} A_{j}^{\omega}=\sum_{k} \beta_{i j}^{k} A_{k}^{\omega} \text { where } \beta_{i j}^{k}:=\sum_{\alpha \in U} \alpha \beta_{i j}^{k}(\alpha)
$$

thus $\mathcal{A}^{\omega}:=\left\langle A_{i}^{\omega}\right\rangle$ is a self-adjoint matrix algebra containing $I$ and we refer to the $\beta_{i j}^{k}$ as the parameters or structure constants of the $\mathcal{A}^{\omega}$. Note that this weighted adjacency algebra is not necessarily closed under the entry-wise product, hence it is not, in general, a coherent algebra. The weighted intersection matrices are defined in the obvious way,

$$
M_{j}:=\left(\beta_{i j}^{k}\right), 0 \leq i, k<r .
$$

Switching equivalent weights have identical parameters and therefore identical intersection matrices. 


\subsection{The fission induced by a weight}

The weighted CC $(\mathfrak{A}, \omega)$ has a natural fission in which $R_{i}$ is partitioned according to distinct values of $\omega$. Put

$$
\left(A_{i}^{\alpha}\right)_{x y}:= \begin{cases}1 & \text { if }\left(A_{i}^{\omega}\right)_{x y}=\alpha \\ 0 & \text { otherwise. }\end{cases}
$$

Some useful properties are:

1. $A_{i}^{\alpha} \circ A_{j}^{\beta}=\delta_{i, j} \delta_{\alpha, \beta} A_{i}^{\alpha}$;

2. $A_{i}=\sum_{\alpha} A_{i}^{\alpha}$;

3. $A_{i}^{\omega}=\sum_{\alpha} \alpha A_{i}^{\alpha}$.

Definition 2.5. ( $\mathfrak{A}, \omega)$ has minimal closure if the fission $\left\{A_{i}^{\alpha}\right\}$ forms a CC.

The terminology draws on the notion of the coherent closure of a set of matrices as the smallest CA containing them (see $[21,28]$ for more). The coherent closure of $(\mathfrak{A}, \omega)$ is the $\mathrm{CC}$ whose $\mathrm{CA}$ is the coherent closure of the matrix algebra $\mathcal{A}^{\omega}$. Clearly

$$
\sum_{i \in \mathcal{L}} A_{i}^{1}=I
$$

and the $A_{i}^{\alpha}$ sum to $J$. Furthermore, by the Hermitian property of the weight,

$$
\left(A_{i}^{\alpha}\right)^{T}=A_{i^{*}}^{\bar{\alpha}}
$$

but the fission is not in general coherent and may in particular generate a matrix algebra of dimension greater than $r t$.

A weighted CC may be represented in a natural way as a $t$-fold cover of the configuration. The main goal of this work is to characterize regular weights on CCs in this way, and to describe the construction of a CC of rank $r t$ - the covering configuration - derived from the cover.

Let $U=U_{t}$ with generator $\zeta$, let $\Gamma$ be a graph or digraph with vertex set $X$, and $\omega$ a weight on $\Gamma$. Following [14], we define the $t$-fold cover of $\Gamma$ afforded by $\omega$ as follows. The vertex set is $X \times\{1,2, \ldots, t\}$. We abuse notation, denoting the $t$ copies of each vertex $x$ by $\left\{x_{1}, x_{2}, \ldots, x_{t}\right\}$. Assign adjacencies by $x_{i} \sim y_{j}$ whenever $x \sim y$ in $\Gamma$ and $\omega(x, y)=\zeta^{i-j}$. The induced permutation of indices, $i \mapsto j$ determines a permutation $\sigma$ of $U$, namely $\zeta^{k} \mapsto \zeta^{k+j-i}$ which is simply multiplication by $\zeta^{j-i}$. Let $Z_{\sigma}$ be the image of $\sigma \in U$ in the left regular representation of $U$ as a multiplicative group. Then $\left\{Z_{\sigma} \mid \sigma \in U\right\}$ is a cyclic group generated by $Z_{\zeta}$ and the element $Z_{\zeta^{k}}$ corresponds to the $k^{\text {th }}$ power of the cycle $(1,2, \ldots, t)$ on indices. Observe that $\sum_{\sigma \in U} Z_{\sigma}$ is the all-ones matrix $J$. Indeed, the $Z_{\sigma}$ are the adjacency matrices of a cyclic group scheme on $t$ points.

Example 2.6. We construct a weight with values in $U_{3}$ on the cycle $C_{3}$, a DRG of diameter 3. The non-trivial basic graphs are shown in Figure 2. Define a weight $\omega$ by:

$$
A_{1}^{\omega}=\left[\begin{array}{cccccc} 
& \alpha & & & & \bar{\alpha} \\
\bar{\alpha} & & \alpha & & & \\
& \bar{\alpha} & & \alpha & & \\
& & \bar{\alpha} & & \alpha & \\
& & & \bar{\alpha} & & \alpha \\
\alpha & & & & \bar{\alpha} &
\end{array}\right], \quad A_{2}^{\omega}=\left[\begin{array}{ccccccc} 
& & \bar{\alpha} & & & \alpha & \\
& & & \bar{\alpha} & & \alpha \\
\alpha & & & & \bar{\alpha} & \\
& \alpha & & & & \bar{\alpha} \\
\bar{\alpha} & & \alpha & & & \\
& \bar{\alpha} & & \alpha & &
\end{array}\right],
$$



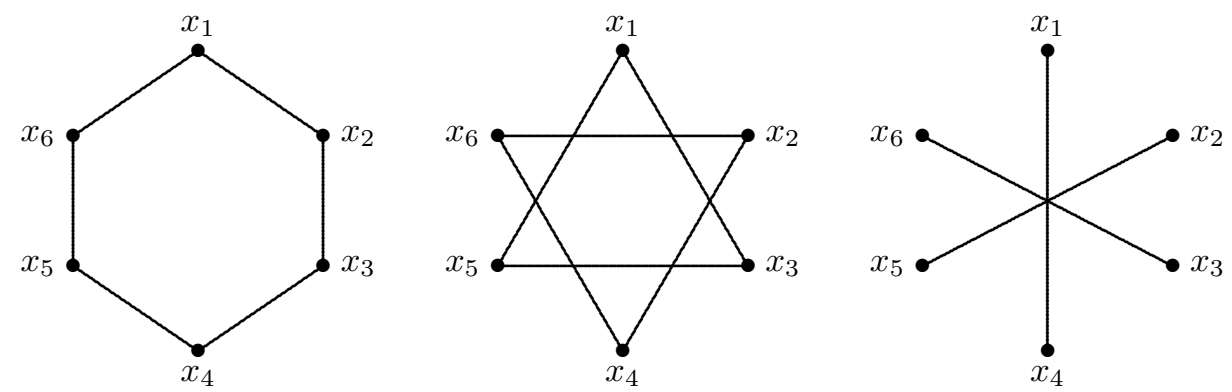

Figure 2: Distance graphs of $C_{3}$.

$$
A_{3}^{\omega}=\left[\begin{array}{llllll} 
& & & 1 & & \\
& & & & 1 & \\
& & & & & 1 \\
1 & & & & & \\
& 1 & & & & \\
& & 1 & & &
\end{array}\right] .
$$

Working out the products, we see that

$$
\begin{aligned}
\left(A_{1}^{\omega}\right)^{2} & =2 I+A_{2}^{\omega}, & \left(A_{2}^{\omega}\right)^{2} & =2 I+A_{2}^{\omega}, \\
A_{1}^{\omega} A_{2}^{\omega}=A_{2}^{\omega} A_{1}^{\omega} & =A_{1}^{\omega}+2 A_{3}^{\omega}, & A_{2}^{\omega} A_{3}^{\omega}=A_{3}^{\omega} A_{2}^{\omega} & =A_{1}^{\omega}, \\
A_{1}^{\omega} A_{3}^{\omega}=A_{3}^{\omega} A_{1}^{\omega} & =A_{2}^{\omega}, & \left(A_{3}^{\omega}\right)^{2} & =I,
\end{aligned}
$$

and therefore the weighted intersection matrices are

$$
M_{1}^{\omega}=\left[\begin{array}{llll}
1 & 0 & 1 & 0 \\
0 & 1 & 0 & 2
\end{array}\right], \quad M_{2}^{\omega}=\left[\begin{array}{llll}
0 & 1 & 0 & 2 \\
2 & 0 & 1 & 0 \\
& 1 & & 1
\end{array}\right], \quad M_{3}^{\omega}=\left[\begin{array}{rrr} 
& & 1 \\
& & 1 \\
1 & &
\end{array}\right] .
$$

Note. Merging the non-trivial relations or, equivalently, summing $A_{i}, i \neq 0$, and also the $A_{i}^{\omega}$, we see that this weight fuses to a regular 3-graph.

\section{Main theorem}

Theorem 3.1. Let $\mathfrak{A}=\left(X,\left\{R_{i}\right\}_{i \in \mathcal{I}}\right)$ be a coherent configuration of rank $r$ on $n:=|X|$ vertices and suppose $\omega$ is a regular weight on $\mathfrak{A}$ with values in $U=U_{t}$. Then $\omega$ induces a rank tr coherent configuration on tn vertices with relations given by

$$
\sum_{\alpha \in U} A_{i}^{\alpha} \otimes Z_{\sigma \alpha} \quad(i \in \mathcal{I}, \sigma \in U)
$$

and parameters $\left\{\beta_{i j}^{k}(\alpha)\right\}$.

Proof. Let $T:=\{1,2, \ldots, t\}$ and let $\Gamma_{i}$ be one of the basic graphs in $\mathfrak{A}$. The $t$-fold cover of $\Gamma_{i}$ that is induced by $\omega$ has vertex set $Y:=X \times T$, and adjacency matrix

$$
\sum_{\alpha \in U} A_{i}^{\alpha} \otimes Z_{\alpha}
$$


Motivated by this, and looking to define the matrices of a CA on $Y$, we put

$$
C_{i, \sigma}:=\sum_{\alpha \in U} A_{i}^{\alpha} \otimes Z_{\sigma \alpha}
$$

for $i \in \mathcal{I}$ and $\sigma \in U$, we claim that $\mathcal{C}:=\left\langle C_{i, \sigma}\right\rangle_{\mathbb{C}}$ is the coherent algebra of a CC $\mathfrak{C}$.

We show that $\mathcal{C}$ satisfies (i)-(iv) of Definition 2.1. We have observed that $\sum_{\sigma \in U} Z_{\sigma}=$ $J$. Since $\sum_{\alpha \in U} A_{i}^{\alpha}=A_{i}$ for all $i$, and $\sum_{i \in \mathcal{I}} A_{i}=J$, we see that

$$
\begin{aligned}
\sum_{i \in \mathcal{I}} \sum_{\sigma \in U} C_{i, \sigma} & =\sum_{i \in \mathcal{I}} \sum_{\sigma \in U} \sum_{\alpha \in U} A_{i}^{\alpha} \otimes Z_{\sigma \alpha} \\
& =\left(\sum_{i \in \mathcal{I}} \sum_{\alpha \in U} A_{i}^{\alpha}\right) \otimes\left(\sum_{\sigma \in U} Z_{\sigma \alpha}\right) \\
& =\left(\sum_{i \in \mathcal{I}} A_{i}\right) \otimes J_{t} \\
& =J_{n} \otimes J_{t} \\
& =J_{n t} .
\end{aligned}
$$

Hence $\mathcal{C}$ satisfies (i).

Let $\mathcal{L} \subseteq \mathcal{I}$ be the unique set of indices such that $\sum_{i \in \mathcal{L}} A_{i}=I_{n}$. (Assume, without loss of generality, that $\mathcal{L}=\{0\}$ if $\mathcal{A}$ is homogeneous.) We claim $I_{n t}=\sum_{i \in \mathcal{L}} C_{i, 1}$. Since $\omega(x, x)=1$ for all $x, A_{i}^{\alpha}=0$ if $i \in \mathcal{L}$ and $\alpha \neq 1$. Consequently, $i \in \mathcal{L}$ implies $A_{i}=A_{i}^{1}$. Hence,

$$
\begin{aligned}
\sum_{i \in \mathcal{L}} C_{i, 1} & =\sum_{i \in \mathcal{L}} \sum_{\alpha} A_{i}^{\alpha} \otimes Z_{\alpha} \\
& =\sum_{i \in \mathcal{L}} A_{i} \otimes Z_{0} \\
& =\left(\sum_{i \in \mathcal{L}} A_{i}\right) \otimes I_{t} \\
& =I_{n} \otimes I_{t}=I_{n t} .
\end{aligned}
$$

This proves that $\mathcal{C}$ satisfies (ii).

The transpose of $M \otimes N$ is $M^{T} \otimes N^{T}$. Since $\omega(y, x)=\overline{\omega(x, y)},\left(A_{i}^{\alpha}\right)^{T}=\left(A_{i^{*}}\right)^{\bar{\alpha}}$. The transpose of a permutation matrix is its matrix inverse, hence $Z_{\sigma}^{T}=Z_{\sigma}^{-1}=Z_{\sigma^{-1}}$. Therefore,

$$
C_{i, \sigma}^{T}=\sum_{\alpha} A_{i^{*}}^{\bar{\alpha}} \otimes Z_{(\sigma \alpha)^{-1}}=\sum_{\bar{\alpha}} A_{i^{*}}^{\bar{\alpha}} \otimes Z_{\bar{\sigma} \bar{\alpha}}=C_{i^{*}, \bar{\sigma}}
$$

thus $\mathcal{C}$ satisfies (iii).

Finally, we obtain the structure constants as follows. We claim:

$$
\left(\sum_{\alpha \in U} A_{i}^{\alpha} \otimes Z_{\sigma \alpha}\right)\left(\sum_{\beta \in U} A_{j}^{\beta} \otimes Z_{\tau \beta}\right)=\sum_{k \in \mathcal{I}} \sum_{\nu \in U} \beta_{i j}^{k}(\nu) \sum_{\gamma \in U}\left(A_{k}^{\gamma} \otimes Z_{\sigma \tau \nu \gamma}\right) .
$$


The left hand side of equation (3.4) is equal to

$$
\begin{aligned}
\sum_{\alpha, \beta \in U}\left(A_{i}^{\alpha} A_{j}^{\beta}\right) \otimes\left(Z_{\sigma \alpha} Z_{\tau \beta}\right) & =\sum_{\alpha, \beta \in U} A_{i}^{\alpha} A_{j}^{\beta} \otimes Z_{\sigma \tau \alpha \beta} \\
& =\sum_{\mu \in U}\left(\sum_{\alpha \beta=\mu} A_{i}^{\alpha} A_{j}^{\beta}\right) \otimes Z_{\sigma \tau \mu}
\end{aligned}
$$

combining terms with the same second tensorand. We now consider the $(x, z)$ entry of each product $A_{i}^{\alpha} A_{j}^{\beta}$ for a fixed $(x, z) \in R_{k}$, setting $\gamma:=\omega(x, z)$. This equals the number of triangles $(x, y, z)$ of type $(i, j, k)$ with weight $\alpha \beta \bar{\gamma}$. Since we are summing these products over all $\alpha$ and $\beta$ with $\alpha \beta=\mu$, we account for all such triangles, and the number of these is $\beta_{i j}^{k}(\alpha \beta \bar{\gamma})$. Thus

$$
\begin{aligned}
\sum_{\mu \in U}\left(\sum_{\alpha \beta=\mu} A_{i}^{\alpha} A_{j}^{\beta}\right) \otimes Z_{\sigma \tau \mu} & =\sum_{\mu \in U}\left(\sum_{\gamma \in U} \sum_{k \in \mathcal{I}} \beta_{i j}^{k}(\mu \bar{\gamma}) A_{k}^{\gamma}\right) \otimes Z_{\sigma \tau \mu} \\
& =\sum_{\mu \in U} \sum_{\gamma \in U} \sum_{k \in \mathcal{I}} \beta_{i j}^{k}(\mu \bar{\gamma})\left(A_{k}^{\gamma} \otimes Z_{\sigma \tau \mu}\right) .
\end{aligned}
$$

Next, observe that $\beta_{i j}^{k}(\nu)$ occurs exactly $t$ times, once for each $\gamma$ with $\mu=\nu \gamma$. Factoring gives

$$
\sum_{k \in \mathcal{I}} \sum_{\nu \in U} \beta_{i j}^{k}(\nu) \sum_{\gamma \in U} A_{k}^{\gamma} \otimes Z_{\sigma \tau \nu \gamma}
$$

which proves the claim. Hence $\beta_{i j}^{k}(\nu)$ is the coefficient of $C_{k, \sigma \tau \nu}$ in the product $C_{i, \sigma} C_{j, \tau}$.

Remark 3.2. If $\mathfrak{A}$ is an association scheme, then $\mathcal{L}=\{0\}$, and $C_{0,1}=I_{n t}$.

Remark 3.3. The following are clear from the proof of Theorem 3.1.

(i) $\mathbb{C}$ is homogeneous if and only if $\mathfrak{A}$ is homogeneous.

(ii) $\mathfrak{C}$ is symmetric if and only if $\mathfrak{A}$ is symmetric and $\omega$ is real-valued, that is, $t=2 . C_{i 1}$ is always symmetric if $A_{i}$ is.

(iii) $\mathfrak{C}$ is commutative if and only if $\mathbb{A}$ is commutative.

(iv) If the $A_{i}^{\alpha}$ form a $\mathrm{CC}$, then we are in the case of minimal closure, and $\mathbb{C}$ is a fusion of a Kronecker product configuration.

(v) The parameter $\beta_{i j}^{k}(\nu)$ in the proof of (iv) clearly does not depend on $\sigma$ or $\tau$. This means that each parameter of $\mathbb{C}$ is duplicated $t^{2}$ times:

$$
p_{i \sigma, j \tau}^{k \sigma \tau \nu}=p_{i 1, j 1}^{k \nu} \quad \forall \sigma, \tau \in U_{t} .
$$

\section{Discussion and analysis}

Example 4.1. This example relates to $\Gamma=\operatorname{SRG}(112,30,2,10)$ which is known by many names in the literature, including the collinearity graph of $\mathrm{GQ}(3,9), \mathrm{O}^{-}(6,3)$, and the 
first sub-constituent of the McLaughlin graph, $\mathrm{McL}_{1}$ to name just three. It has a strongly regular decomposition into two Gewirtz graphs $(\operatorname{SRG}(56,10,0,2))$ [7].

Let $\mathfrak{A}$ be the rank 3 scheme afforded by $\Gamma$. We construct a regular weight on $\mathfrak{A}$ with values in $U_{2}$, making use of the decomposition. Let $X_{1}$ and $X_{2}$ be the two sets of 56 vertices. Define $\omega(x, y)$ for $x \neq y$ to be -1 when $x$ and $y$ are in the same half of this partition, and +1 otherwise. Note that $\omega$ restricted to either Gewirtz graph is a trivial weight with matrix $2 I-J$.

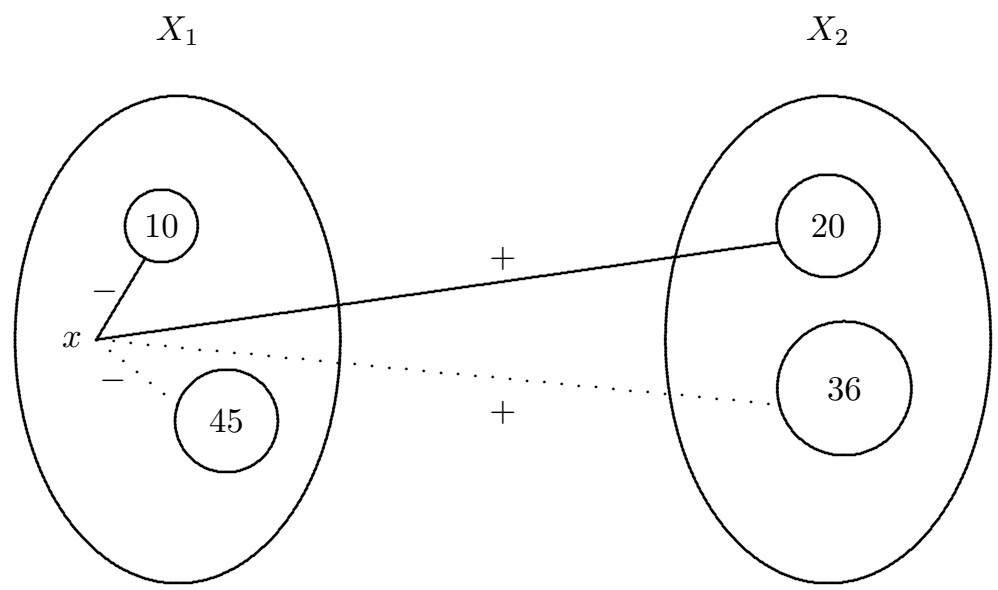

Figure 3: Strongly regular decomposition of $\operatorname{SRG}(112,30,2,10)$.

In Figure 3, a solid line indicates adjacency in $\Gamma$, a dotted line non-adjacency. This weighted SRG has minimal closure, since the $A_{i}^{\alpha}$ form a rank 5 scheme, in fact a strongly regular design or SRD ([13]). Since $\omega(x, y)$ is determined by the parity of $\{x, y\} \cap X_{1}$, the four non-trivial relations are given by the four combinations of attributes: adjacency/nonadjacency, and this parity. There are many related configurations. For example, another copy of the Gewirtz graph may be adjoined to construct an example of triality ([15]). These 112 vertices form the first subconstituent of the McLaughlin graph; the second subconstituent also admits a strongly regular decomposition ([3]).

Some interesting properties of this example:

1. Minimal closure is rare (see [21]).

2. The SRD is cometric, but not metric, which is also rare.

3. The covering configuration $\mathbb{C}$ is also cometric, but not metric, having rank 6 on 224 points. This example arises as the Q-bipartite double of $\mathrm{McL}_{1}$ (see [18]).

The Gewirtz graph admits a non-trivial regular weight with values in $U_{4}$, constructed via a monomial representation of $2 . \mathrm{L}_{3}(4)$ ([20]). The covering configuration is neither metric nor cometric, has rank 12 on 224 vertices, and contains the doubled Gewirtz graph (DRG $[10,9,8,2,1 ; 1,2,8,9,10])$ as a quotient. 


\subsection{Intersection matrices}

Lemma 4.2. The intersection matrices of $\mathcal{C}$ have the form $M_{j \tau}=\sum_{\nu \in U_{t}} M_{j}^{\nu} \otimes Z_{\tau \nu}$ where $\left[M_{j}^{\nu}\right]_{i k}:=\beta_{i j}^{k}(\nu)$

Proof. We may assume the relations $C_{i \sigma}$ are ordered lexicographically, that is first by $i$ and then by $\sigma \in\left\{1, \zeta, \ldots, \zeta^{t-1}\right\}$ so that the intersection matrix $M_{j \tau}$ has $(i, k)$ block given by $\left(p_{i \sigma, j \tau}^{k \sigma \tau \nu}\right)_{\sigma, \sigma \tau \nu}$. By equation (3.6) this block has the value $\beta_{i j}^{k}(\nu)$ in position $(\sigma, \sigma \tau \nu)$ which means that it has the form $\sum_{\nu} \beta_{i j}^{k}(\nu) Z_{\tau \nu}$. Hence $M_{j \tau}$ is the required sum of Kronecker products.

Lemma 4.3. Let $\omega$ be a regular weight on the cc $\mathfrak{A}$, and let $\widetilde{\omega}$ be an equivalent weight obtained by switching $\omega$ by a factor of $\tau=\zeta^{l}$ at vertex $x$. Further let $\mathbb{C}=\left(Y,\left\{C_{i \sigma}\right\}\right)$ and $\widetilde{\mathbb{C}}=\left(Y,\left\{\widetilde{C}_{i \sigma}\right\}\right)$ be the covering configurations induced by $\omega$ and $\widetilde{\omega}$ respectively. Then $\widetilde{\mathbb{C}}$ is obtained from $\mathbb{C}$ by permuting $\left\{x_{i}\right\}$ according to the permutation $(1,2, \ldots, t)^{l}$ resulting from multiplication by $\tau$ on $U$.

Proof. Suppose $\omega(x, y)=\alpha$. For some $i$ and $j,\left(x_{1}, y_{j}\right) \in C_{i 1}$ of $\mathcal{C}$, thus $\alpha=\zeta^{j-1}$. Now, $\widetilde{\omega}(x, y)=\tau \alpha$ by assumption, so we have $\left(x_{1-l}, y_{j}\right) \in \widetilde{C}_{i 1}$. But this implies that $\widetilde{\mathbb{C}}$ is obtained from $\mathbb{C}$ by the permutation $x \mapsto x_{1-l}$, which corresponds to multiplication by $\tau$ on $U$.

\subsection{Special cases}

(i) If $\omega$ has minimal closure, $\mathbb{C}$ is a fusion of a tensor product of two CCs.

(ii) If $\omega$ is trivial in the sense that $A_{i}^{\alpha}=0$ for all but one value of $\alpha, \omega$ has minimal closure, and $\mathcal{C}=\mathcal{A}^{\omega} \otimes Z$.

(iii) If $\mathfrak{A}$ has rank $2\left(\omega\right.$ is regular on $K_{n}$ ), $\mathbb{C}$ is a $t$-fold cover of $K_{n}$. It is not necessarily distance regular. This case encompasses the regular two-graphs $(t=2)$, and the regular 3-graphs $(t=3)$ of Higman [9] and Kalmanovich [16].

(iv) If $t=2, \mathfrak{A}$ is a (symmetric) scheme, and $\mathcal{A}^{\omega}$ has minimal closure (say $\mathfrak{b}$, where $\left.\mathfrak{s}=\left(X,\left\{B_{i}\right\}\right)\right)$, then the covering configuration is isomorphic to the extended Qbipartite double of $\mathfrak{i}$, when it exists, if the rank of $\mathfrak{b}$ is odd $([18,3.1])$. Existence requires $\mathfrak{b}$ to be cometric with an additional condition on the Krein parameters. For even rank, the covering configuration has a fusion (merging just two classes) that is isomorphic to the extended Q-bipartite double, provided that there is exactly one class of $\mathfrak{A}$ on which $\omega$ is constant. Note that a minimal closure of a weight with values in $U_{2}$ has even rank only when the weight is constant on an odd number of classes of A. The isomorphism is $M \otimes N \mapsto N \otimes M$ on the $C_{i \sigma}$ of the cover configuration.

\subsubsection{Necessary conditions for a covering configuration}

In the case of commutative CCs we extend [16, Prop. 5.4] in a natural way, as follows.

Let $\mathbb{C}=\left(X,\left\{R_{i}\right\}\right)$ be a commutative $\mathrm{CC}$ of rank $t r$ such that the first $t$ intersection matrices have the form $M_{j}=I_{r} \otimes Z_{\zeta^{j}}$, for $0 \leq j<t$, and let $U=\langle\zeta\rangle$ the group of roots of unity of order $t$. Index the relations according to the $r$ blocks of size $t$, so that

$$
C_{i, \zeta^{k}}=R_{i t+k}
$$


and suppose that for any $i, j, k$ and $\nu$ :

$$
p_{i \sigma, j \tau}^{k \sigma \tau \nu}=p_{i 1, j 1}^{k \nu}
$$

for all $\sigma$ and $\tau$ in $U$. We intend to show that under these conditions, $\mathbb{C}$ must arise as the covering configuration of a regular weight on a quotient of $\mathbb{C}$.

Lemma 4.4. If $j<t$ and $p_{i j}^{k} \neq 0$, then $k=i+j(\bmod t)$; in particular, $i$ and $k$ lie in the same block of $M_{j}$.

Proof. This follows from $M_{j}=I \otimes Z_{\zeta^{j}}$.

Observe that $E:=\cup_{j=0}^{t-1} R_{j}$ is a parabolic in the sense of [10]. Indeed, $M_{0}=I_{r t}$ implies that $R_{0}$ is the identity relation of $\mathbb{C}$. Further, $E$ is symmetric, since $(x, y) \in R_{i}$ for $i<t$ implies that $p_{i^{*} i}^{0} \neq 0$, so $i^{*}$ is in the same block of $M_{i}$ as 0 . That is, $(y, x) \in E$. Given $(x, y) \in R_{i}$ and $(y, z) \in R_{j}$ with $0 \leq i, j<t$, we see that $(x, z) \in R_{k}$ for some $k<t$, because $k$ must lie in the same block of $M_{j}$ as $i$, since all non-diagonal blocks are zero. Hence, $E$ is a transitive relation.

As a parabolic, $E$ induces an equivalence relation on the indices: If there exist $x, x^{\prime}$, $y, y^{\prime} \in X$ such that $\left(x, x^{\prime}\right) \in E,\left(y, y^{\prime}\right) \in E,(x, y) \in R_{i}$ and $\left(x^{\prime}, y^{\prime}\right) \in R_{j}$, then $i \sim j$. Write $[i]$ for the equivalence class of $i$. In addition, the parabolic affords a quotient (homogeneous) configuration $\mathfrak{A}:=\left(\bar{X},\left\{\bar{R}_{[i]}\right\}\right)$ with an associated partition of the vertex set $X$ into fibres of size $t$. The fibre containing $x$ is

$$
[x]=\{y \mid(x, y) \in E\} .
$$

We will henceforth suppress the bracket notation for fibres, writing $x=\left\{x_{1}, x_{2}, \ldots x_{t}\right\}$.

For $j \in[0]$, Lemma 4.4 implies that $p_{k j}^{k}=0$ for $j \neq 0$. But then $R_{k}$ restricted to $x \times y$ has valency at most 1 . We conclude that the number of relations occurring between any two fibres is $t$. We have: For $k \in \mathcal{I}$ and $x \in X$,

$$
|[k]|=|x|=t
$$

Denoting the graph of $R_{j}$ by $\Gamma_{j}$, we have proved the following:

Lemma 4.5. For all $j \notin[0], \Gamma_{j}$ is a t-fold cover of $\Gamma_{[j]}$.

Corollary 4.6. The natural partition of $\mathcal{I}$ according to blocks of $M_{j}$, for $0 \leq j<t$ is the same as that determined by the equivalence classes of the parabolic. That is,

$$
[m t]=\{m t, m t+1, \ldots, m t+t-1\} .
$$

Proof. Suppose $j \in[i]$ so that there exist $x_{1}, x_{2}, y_{1}, y_{2} \in X$ with $\left(x_{1}, y_{1}\right) \in R_{i}$ and $\left(x_{2}, y_{2}\right) \in R_{j}$. Then, by the discussion above, $\left(x_{1}, y_{3}\right) \in R_{j}$ for some $y_{3} \in y$ and therefore $p_{i k}^{j} \neq 0$ for some $k<t$.

But then $j=i+k(\bmod t)$ by Lemma 4.4.

Recall that $C_{0, \zeta^{k}}=R_{k}$ for $k<t, C_{0, \sigma}$ has intersection matrix $I_{r} \otimes Z_{\sigma}$, and $C_{m, 1}=R_{m t}$ for $0 \leq m<r$. Fix a fibre $a$ (from here on), and order it so that $\left(a_{i}, a_{i+1}\right) \in C_{0 \zeta}$, for each $i$, with addition modulo $t$. This ensures that the perfect matching induced on $a$ corresponds 
to the permutation $(1,2, \ldots, t)$ on indices, which in turn corresponds to the permutation of $U$ induced by multiplication by $\zeta$.

For each $x \in \bar{X},(a, x) \in \bar{R}_{[m t]}$ for some $m$. Order $x$ so that $\left(a_{j}, x_{j}\right) \in C_{m, 1}$. In what follows, we mix the notations regarding indexation of the relations of $\mathbb{C}$. Where two indices are given, we refer to $C_{i, \sigma}$ as above; where one index is given we refer to the original numbering of the relations.

Lemma 4.7. With notation as above, $\left(x_{i}, x_{i+1}\right) \in C_{0, \zeta}$ for all $x \in \bar{X}$.

Proof. For some $\sigma,\left(x_{i}, x_{i+1}\right) \in C_{0, \sigma} ;\left(a_{i}, x_{i+1}\right) \in R_{l}$ for some $l$, and $\left(a_{i}, x_{i}\right) \in R_{m 1}$ for some $m$. Note that $l \in[m]$. Since $a_{i}, a_{i+1}$, and $x_{i+1}$ form a triangle of type $(0 \zeta, m 1, l)$,
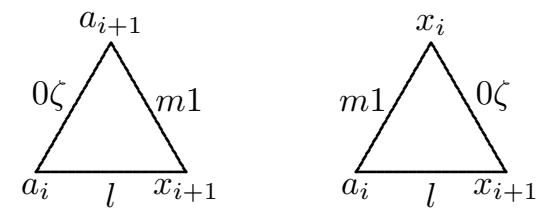

Figure 4: Triangles $\left(a_{i}, a_{i+1}, x_{i+1}\right)$ and $\left(a_{i}, x_{i}, x_{i+1}\right)$.

we see that $p_{0 \zeta, m 1}^{l} \neq 0$. Since $\mathbb{C}$ is commutative, $R_{l}=C_{m \zeta}$ by Lemma 4.4. Now observe that $a_{i}, x_{i}$, and $x_{i+1}$ form a triangle of type $(m 1,0 \sigma, m \zeta)$, and therefore $\sigma=\zeta$.

Next, following [16] we show that all matchings are cyclic.

Lemma 4.8. With notation as above, all matchings between fibres of $\mathbb{C}$ are cyclic.

Proof. Suppose that $\left(x_{i}, y_{j}\right) \in R_{k}$ and $\left(x_{i+1}, y_{l}\right) \in R_{k}$. We must show that $l=j+1$. The triangle $\left(x_{i}, x_{i+1}, y_{j}\right)$ has type $(1, m, k)$ for some $m$, indicating that $p_{1 m}^{k} \neq 0$. As in the previous lemma, this implies that $k=m+1$. On the other hand, the triangle $\left(x_{i+1}, y_{l-1}, y_{l}\right)$ has type $(b, 1, k)$ for some $b$, hence $k=b+1$. But then $m=b$, and by Lemma $4.5, y_{l-1}=y_{j}$ as desired.

Corollary 4.9. For all $x \in X,\left(x_{i}, x_{i+k}\right) \in R_{k}$, thus $R_{k}$ induces on each fibre the perfect matching corresponding to the $k^{\text {th }}$ power of the cycle $(1,2, \ldots, t)$.

Proof. The result follows by Lemma 4.7 and induction (on $k$ ) applied to the triangles $\left(x_{i-k}, x_{i}, x_{i+1}\right)$.

Lemma 4.10. For $x \in X,\left(a_{i}, x_{i+k}\right) \in R_{m t+k}$ for $0 \leq k<t$.

Proof. The case $k=0$ holds by choice of ordering of $x$. Induction applied to the triangles $\left(a_{i}, x_{i+k-1}, x_{i+k}\right)$ gives the desired result.

We now define a weight on $\mathfrak{A}$ by means of $C_{i 1}$. Let $x, y \in \bar{X}$ and suppose $(x, y) \in \bar{R}_{[j]}$. Then $C_{j, 1}$ provides a cyclic matching between $x$ and $y$ corresponding to, say, $\alpha \in U$. Set $\omega(x, y):=\alpha$. Observe that $\omega(a, x)=1$ for all $x$.

The next lemma shows how to determine the weight of an edge in $\bar{\Gamma}_{[i]}$ from any edge in $\Gamma_{i}$. 
Lemma 4.11. If $\left(x_{i}, y_{j}\right) \in C_{k \sigma}$, then $\omega(x, y)=\bar{\sigma} \zeta^{j-i}$.

Proof. Consider $\left(x_{i}, y_{j}\right) \in C_{k, \sigma}$. Let $l$ be such that $\left(x_{i}, y_{l}\right) \in C_{k 1}$ and note that the triangle $\left(x_{i}, y_{l}, y_{j}\right)$ has type $\left(k 1,0 \zeta^{j-l}, k \sigma\right)$. By Proposition 4.6, $\sigma=\zeta^{j-l}$. This implies that $\left(x_{i}, y_{l+m}\right) \in C_{k, \zeta^{m}}$. We conclude that the matching between $x$ and $y$ in $C_{k, \sigma}$ is $\alpha \sigma$, where $\alpha=\omega(x, y)$.

We now prove the second main result which is the extension of [16, Prop. 5.4].

Theorem 4.12. Let $\mathbb{C}=\left(X,\left\{R_{i}\right\}\right)$ be a commutative $C C$ of rank rt with the first $t$ intersection matrices given by

$$
M_{j}=I_{r} \otimes Z_{\zeta^{j}} \quad 0 \leq j<t,
$$

where $U=U_{t}=\langle\zeta\rangle$ is the group of roots of unity of order $t$. Label the relations according to the blocking of $M_{j}$ :

$$
C_{i, \zeta^{k}}:=R_{i t+k} \quad 0 \leq i<r, 0 \leq k<t
$$

and suppose that the CC parameters satisfy, for any $i, j, k$ and $\nu$ :

$$
p_{i \sigma, j \tau}^{k \sigma \tau \nu}=p_{i 1, j 1}^{k \nu}
$$

for all $\sigma$ and $\tau$ in $U$. Then $\mathbb{C}$ arises as the covering configuration (in the sense of Theorem 3.1) from a regular weight $\omega$ on the quotient scheme $\mathfrak{A}=\mathbb{C} / E$.

Proof. From the discussion and lemmas above, what remains to be shown is that $\omega$ is regular on the quotient configuration $\overline{\mathbb{C}}=\left(\bar{X},\left\{\bar{R}_{[i]}\right\}\right)$. Let $(x, z) \in \bar{R}_{[k]}$. We consider all $y$ such that $(x, y, z)$ has type $(i, j, k)$ and weight $\nu$. Let $l$ be such that $\left(x_{1}, z_{l}\right) \in C_{k \nu}$. If $(x, y) \in \bar{R}_{[i]}$ and $(y, z) \in \bar{R}_{[j]}$, then $\left(x_{1}, y_{m}\right) \in C_{i 1}$ for some $m$, and this determines (exactly one) $\tau$ with $\left(y_{m}, z_{l}\right) \in C_{j \tau}$. By Lemma 4.11,

$$
\begin{aligned}
\delta \omega(x, y, z) & =\omega(x, y) \omega(y, z) \overline{\omega(x, z)} \\
& =\zeta^{m-1} \bar{\tau} \zeta^{l-m} \nu \zeta^{1-l} \\
& =\bar{\tau} \nu
\end{aligned}
$$

from which we see that triangles of weight $\nu$ occur exactly when $\tau=1$. These triangles are counted by the parameter $p_{i 1, j 1}^{k \nu}$ which is independent of the choice of $x_{1}$ and $z_{l}$.

Note that in the proof above we are counting distinct $y$, and that for each $y$ there is exactly one $y_{m}$ as indicated. Thus we may use $C_{i 1}$ without loss of generality, since $\left(x_{1}, y_{m}\right) \in C_{i \sigma}$ would yield the same result. In fact triangles of type $(i \sigma, j \tau, k \nu)$ will have weight $\nu$ exactly when $\sigma \tau=1$, which is expected as in that case $p_{i \sigma, j \tau}^{k \nu}=p_{i 1, j 1}^{k \nu}$

\section{Examples}

\subsection{A rank 12 scheme on 18 points}

The covering configuration of Example 2.6 has rank $12(=4 \cdot 3)$ on $18(=6 \cdot 3)$ points. It is isomorphic to as18[88] on Hanaki and Izumi's list ([8]). 


\subsection{A family of CCs from regular weights on $H(n, 2)$ with values in $U_{4}$}

This construction is due to Ada Chan (personal communication). We define a regular weight on the Hamming Scheme $H(n, 2)$ with values in $U_{4}$ with generator i. Let $\mathbf{t}$ be an indeterminate, and $K$ the 2 by 2 matrix $\left[\begin{array}{cc}0 & 1 \\ -1 & 0\end{array}\right]$. Form $(I+\mathbf{t} K)^{\otimes n}$, a polynomial in $\mathbf{t}$ with coefficients in the ring of matrices $M_{2,2}(\mathbb{R})^{\otimes n} \simeq M_{2^{n}, 2^{n}}(\mathbb{R})$. Now let $A_{k}^{\omega}$ be the coefficient of $\mathbf{t}^{k}$, scaled by a factor of $\mathbf{i}^{k} \in U_{4}$. We claim this is a regular weight on the Hamming scheme. Indeed, replacing $\mathbf{i}$ with 1 and $K$ with $J-I$ in this process yields the adjacency matrices of the Hamming scheme, with the standard P-polynomial ordering. Noting that $K^{2}=-I$ it is straight-forward to see that $\operatorname{Span}\left(A_{k}^{\omega}\right)$ is coherent. For regularity, we note that $p_{i j}^{k}$ is nonzero only when $i+j+k$ is even, and this implies $\beta_{i j}^{k}( \pm \mathbf{i})=0$ for all $i, j, k$. Proposition 1 of [21] applies, and we conclude that $\omega$ is regular.

The covering configuration induced by this weight is a rank $4(n+1) \mathrm{CC}$ on $2^{n+2}$ vertices. There is a fusion to regular 4 -graph, which is easily seen: replace $\mathbf{t}$ by $\mathbf{i}$, setting

$$
\widetilde{\omega}:=(I+\mathbf{i} K)^{\otimes n},
$$

then verify directly that $\widetilde{\omega}^{2}=2^{n} \widetilde{\omega}$ thus $\widetilde{\omega}$ is the matrix of a regular 4-graph. The covering configuration of $\widetilde{\omega}$ has rank 8 and is symmetric, but not necessarily distance regular.

For $n=2$, the weight is given by:

$$
A_{1}^{\omega}=\left[\begin{array}{cccc}
0 & i & i & 0 \\
-i & 0 & 0 & i \\
-i & 0 & 0 & i \\
0 & -i & -i & 0
\end{array}\right] \quad \text { and } \quad A_{2}^{\omega}=\left[\begin{array}{cccc}
0 & 0 & 0 & 1 \\
0 & 0 & -1 & 0 \\
0 & -1 & 0 & 0 \\
1 & 0 & 0 & 0
\end{array}\right]
$$

The rank 12 covering configuration has color matrix $\left(\sum i A_{i}\right)$ below.

$$
\left[\begin{array}{cccccccccccccccc}
0 & 1 & 2 & 3 & 7 & 4 & 5 & 6 & 7 & 4 & 5 & 6 & 8 & 9 & 10 & 11 \\
3 & 0 & 1 & 2 & 6 & 7 & 4 & 5 & 6 & 7 & 4 & 5 & 11 & 8 & 9 & 10 \\
2 & 3 & 0 & 1 & 5 & 6 & 7 & 4 & 5 & 6 & 7 & 4 & 10 & 11 & 8 & 9 \\
1 & 2 & 3 & 0 & 4 & 5 & 6 & 7 & 4 & 5 & 6 & 7 & 9 & 10 & 11 & 8 \\
5 & 6 & 7 & 4 & 0 & 1 & 2 & 3 & 10 & 11 & 8 & 9 & 7 & 4 & 5 & 6 \\
4 & 5 & 6 & 7 & 3 & 0 & 1 & 2 & 9 & 10 & 11 & 8 & 6 & 7 & 4 & 5 \\
7 & 4 & 5 & 6 & 2 & 3 & 0 & 1 & 8 & 9 & 10 & 11 & 5 & 6 & 7 & 4 \\
6 & 7 & 4 & 5 & 1 & 2 & 3 & 0 & 11 & 8 & 9 & 10 & 4 & 5 & 6 & 7 \\
5 & 6 & 7 & 4 & 10 & 11 & 8 & 9 & 0 & 1 & 2 & 3 & 7 & 4 & 5 & 6 \\
4 & 5 & 6 & 7 & 9 & 10 & 11 & 8 & 3 & 0 & 1 & 2 & 6 & 7 & 4 & 5 \\
7 & 4 & 5 & 6 & 8 & 9 & 10 & 11 & 2 & 3 & 0 & 1 & 5 & 6 & 7 & 4 \\
6 & 7 & 4 & 5 & 11 & 8 & 9 & 10 & 1 & 2 & 3 & 0 & 4 & 5 & 6 & 7 \\
8 & 9 & 10 & 11 & 5 & 6 & 7 & 4 & 5 & 6 & 7 & 4 & 0 & 1 & 2 & 3 \\
11 & 8 & 9 & 10 & 4 & 5 & 6 & 7 & 4 & 5 & 6 & 7 & 3 & 0 & 1 & 2 \\
10 & 11 & 8 & 9 & 7 & 4 & 5 & 6 & 7 & 4 & 5 & 6 & 2 & 3 & 0 & 1 \\
9 & 10 & 11 & 8 & 6 & 7 & 4 & 5 & 6 & 7 & 4 & 5 & 1 & 2 & 3 & 0
\end{array}\right]
$$

The regular 4-graph $\widetilde{\omega}:=I+A_{1}^{\omega}+A_{2}^{\omega}$ satisfies $\widetilde{\omega}^{2}=4 I$. The covering configuration of $\widetilde{\omega}$ has rank 8 and may also be obtained through fusion of the rank 12 above.

In summary, this construction gives regular weights with values in $U_{4}$ on the Hamming Schemes $\mathrm{H}(n, 2)$. These have rank $n+1$ on $2^{n}$ vertices. The covering configurations thus 
have rank $4(n+1)$ on $2^{n+2}$ vertices. These weights fuse to regular 4-graphs always, and the covering configurations of those have rank 8 . In examples constructed to date, the covering configurations are not metric, nor are their symmetrizations, and they are not cometric.

\subsection{CCs afforded by groups}

A CC may have relations determined by the orbitals of a group $G$ acting on a set $X$, in which the centralizer algebra of the natural permutation representation is the coherent algebra $\mathcal{A}$. In this case, a regular weight may exist such that $\mathcal{A}^{\omega}$ is the centralizer algebra of a monomial representation of $G$, induced from a linear representation of a point stabilizer ([14]).

For example, the rank 3 scheme containing the Petersen graph is afforded by the action of $A_{5}$ on 2-sets from $\{1,2,3,4,5\}$. The stabilizer of $\{1,2\}$ is a group $H \simeq S_{3}$, containing $A:=\langle 3,4,5\rangle$ as a subgroup of index 2. This index determines that the monomial representation will afford a weight with values in $U_{2}$. Defining the linear representation

$$
\phi: H \rightarrow C_{2} \text { by } \phi(g)= \begin{cases}1 & g \in A, \\ -1 & g \notin A,\end{cases}
$$

the induced representation $M:=\left.\phi\right|_{H} ^{G}$ is a monomial representation of $G$. The $M(g)$ for $g \in G$ are signed permutation matrices. The centralizer algebra of $M, \mathcal{A}^{\omega}$, defines a regular weight on the Petersen graph.

This construction can be done in general when the point stabilizer $H$ has a normal subgroup $A$ of index $t$, such that $H / A \simeq C_{t}$. The monomial representation induced may or may not afford a nontrivial regular weight on the underlying $\mathrm{CC}$.

In this example, the covering configuration $\mathbb{C}$ is a rank 6 scheme on 20 points, in fact the unique (antipodal, non-bipartite) distance-regular graph DRG $\{3,2,1,1,1 ; 1,1,1,2,3\}$, that is the dodecahedron graph. (It is not the bipartite double of the Petersen graph, which is $\operatorname{DRG}\{3,2,2,1,1 ; 1,1,2,2,3\}$.)

We obtain a permutation representation from $M$, via

$$
M(g) \mapsto M^{+}(g) \otimes Z_{1}+M^{-}(g) \otimes Z_{2}
$$

where $M^{+}, M^{-}, Z_{1}$ and $Z_{2}$ are defined as in Section 2. It is natural to ask whether $\mathcal{C}$ is the centralizer algebra of this permutation representation. In fact, $\mathcal{C}$ is properly contained in this centralizer algebra. It affords a $C C$ with valencies $1,1,3,3,3,3,3,3$ which has a fusion to $\mathbb{C}$. The group affording $\mathbb{C}$ is $A_{5} \times C_{2}$, an extension of our group $G$ by the cyclic $C_{2}$, the latter generated by the even permutation interchanging each $x_{1}$ and $x_{2}$. This is of course the symmetry group of the dodecahedron and is not isomorphic to $S_{5}$.

\section{References}

[1] E. Bannai and T. Ito, Algebraic Combinatorics I: Association Schemes, The Benjamin/Cummings Publishing Company, Menlo Park, California, 1984.

[2] A. E. Brouwer, A. M. Cohen and A. Neumaier, Distance-Regular Graphs, volume 18 of Ergebnisse der Mathematik und ihrer Grenzgebiete, Springer-Verlag, Berlin, 1989, doi: 10.1007/978-3-642-74341-2.

[3] P. J. Cameron, J. M. Goethals and J. J. Seidel, Strongly regular graphs having strongly regular subconstituents, J. Algebra 55 (1978), 257-280, doi:10.1016/0021-8693(78)90220-x. 
[4] P. J. Cameron and J. H. van Lint, Designs, Graphs, Codes and their Links, volume 22 of London Mathematical Society Student Texts, Cambridge University Press, Cambridge, 1991, doi:10.1017/cbo9780511623714.

[5] C. D. Godsil, Algebraic Combinatorics, Chapman and Hall Mathematics Series, Chapman \& Hall, New York, 1993.

[6] C. D. Godsil and A. D. Hensel, Distance regular covers of the complete graph, J. Combin. Theory Ser. B 56 (1992), 205-238, doi:10.1016/0095-8956(92)90019-t.

[7] W. H. Haemers and D. G. Higman, Strongly regular graphs with strongly regular decomposition, Linear Algebra Appl. 114-115 (1989), 379-398, doi:10.1016/0024-3795(89)90471-0.

[8] A. Hanaki and I. Miyamoto, Classification of association schemes with small vertices, http: //math.shinshu-u.ac.jp/ hanaki/as/ (accessed in August 2015).

[9] D. G. Higman, A note on regular 3-graphs, unpublished draft (7 pages).

[10] D. G. Higman, The parabolics of a semi-coherent configuration, unpublished draft (18 pages).

[11] D. G. Higman, Coherent configurations I: Ordinary representation theory, Geometriae Dedicata 4 (1975), 1-32, doi:10.1007/bf00147398.

[12] D. G. Higman, Coherent algebras, Linear Algebra Appl. 93 (1987), 209-239, doi:10.1016/ s0024-3795(87)90326-0.

[13] D. G. Higman, Strongly regular designs and coherent configurations of type $\left[\begin{array}{ll}3 & 2 \\ & 3\end{array}\right]$, Eur. J. Combin. 9 (1988), 411-422, doi:10.1016/s0195-6698(88)80072-6.

[14] D. G. Higman, Weights and t-graphs, Bull. Soc. Math. Belg. Sér. A 42 (1990), 501-521.

[15] D. G. Higman, Rank 5 association schemes and triality, Linear Algebra Appl. 226-228 (1995), 197-222, doi:10.1016/0024-3795(95)00102-w.

[16] D. Kalmanovich, On D. G. Higman's note on regular 3-graphs, Ars Math. Contemp. 6 (2013), 99-115, http://amc-journal.eu/index.php/amc/article/view/243.

[17] M. Klin, A. Munemasa, M. Muzychuk and P.-H. Zieschang, Directed strongly regular graphs obtained from coherent algebras, Linear Algebra Appl. 377 (2004), 83-109, doi:10.1016/j.laa. 2003.06.020.

[18] W. J. Martin, M. Muzychuk and J. Williford, Imprimitive cometric association schemes: Constructions and analysis, J. Algebraic Combin. 25 (2007), 399-415, doi:10.1007/ s10801-006-0043-2.

[19] W. J. Martin and H. Tanaka, Commutative association schemes, Eur. J. Combin. 30 (2009), 1497-1525, doi:10.1016/j.ejc.2008.11.001.

[20] A. D. Sankey, Regular weights of full rank on strongly regular graphs, Israel J. Math. 95 (1996), 1-23, doi:10.1007/bf02761032.

[21] A. D. Sankey, Weighted association schemes, fusions, and minimal coherent closures, J. Algebraic Combin. 41 (2015), 785-815, doi:10.1007/s10801-014-0553-2.

[22] J. J. Seidel, A survey of two-graphs, in: Colloquio Internazionale sulle Teorie Combinatorie (Rome, Italy, 1973), Tomo I, Accademia Nazionale dei Lincei, Rome, volume 17 of Atti dei Convegni Lincei, pp. 481-511, 1976.

[23] J. J. Seidel, More about two-graphs, in: J. Nešetřil and M. Fiedler (eds.), Fourth Czechoslovakian Symposium on Combinatorics, Graphs and Complexity (Prachatice, 1990), NorthHolland, Amsterdam, volume 51 of Annals of Discrete Mathematics, pp. 297-308, 1992, doi: 10.1016/s0167-5060(08)70646-0. 
[24] J. J. Seidel and D. E. Taylor, Two-graphs, a second survey, in: L. Lovász and V. T. Sós (eds.), Algebraic Methods in Graph Theory, Volume II, North-Holland, Amsterdam, volume 25 of Colloquia Mathematica Societatis János Bolyai, pp. 689-711, 1981.

[25] D. E. Taylor, Regular 2-graphs, Proc. London Math. Soc. 35 (1977), 257-274, doi:10.1112/ plms/s3-35.2.257.

[26] D. E. Taylor and R. Levingston, Distance-regular graphs, in: D. A. Holton and J. Seberry (eds.), Combinatorial Mathematics, Springer, Berlin, volume 686 of Lecture Notes in Mathematics, 1978 pp. 313-323, proceedings of the International Conference on Combinatorial Theory (Australian National University, Canberra, 16-27 August 1977).

[27] E. R. van Dam, J. H. Koolen and H. Tanaka, Distance-regular graphs, 2016, arXiv:1410.6294 [math.CO].

[28] B. Yu. Weisfeiler and A. A. Lehman, Reduction of a graph to a canonical form and an algebra which appears in the process, Nauchno-Tekhnicheskaya Informatsiya Ser. 29 (1968), 12-16. 